ARTICLE

\title{
Ultra-high open-circuit voltage of tin perovskite solar cells via an electron transporting layer design
}

\author{
Xianyuan Jiang (1) 1,3, Fei Wang (1) 1,3, Qi Wei (1) 1, Hansheng Li (1) 1, Yuequn Shang (1) 1, Wenjia Zhou (1) 1, \\ Cheng Wang ${ }^{1,2}$, Peihong Cheng (10 ${ }^{1}$, Qi Chen (1) ${ }^{2}$, Liwei Chen (1D ${ }^{2}$ \& Zhijun Ning ${ }^{1 凶}$
}

Tin perovskite is rising as a promising candidate to address the toxicity and theoretical efficiency limitation of lead perovskite. However, the voltage and efficiency of tin perovskite solar cells are much lower than lead counterparts. Herein, indene- $\mathrm{C}_{60}$ bisadduct with higher energy level is utilized as an electron transporting material for tin perovskite solar cells. It suppresses carrier concentration increase caused by remote doping, which significantly reduces interface carriers recombination. Moreover, indene- $C_{60}$ bisadduct increases the maximum attainable photovoltage of the device. As a result, the use of indene- $\mathrm{C}_{60}$ bisadduct brings unprecedentedly high voltage of $0.94 \mathrm{~V}$, which is over $50 \%$ higher than that of $0.6 \mathrm{~V}$ for device based on [6,6]-phenyl-C61-butyric acid methyl ester. The device shows a record power conversion efficiency of $12.4 \%$ reproduced in an accredited independent photovoltaic testing lab.

\footnotetext{
${ }^{1}$ School of Physical Science and Technology, ShanghaiTech University, Shanghai 201210, China. ${ }^{2}$ i-Lab, CAS Center for Excellence in Nanoscience, Suzhou Institute of Nano-Tech and Nano-Bionics, Chinese Academy of Sciences, Suzhou 215123, China. ${ }^{3}$ These authors contributed equally: Xianyuan Jiang,

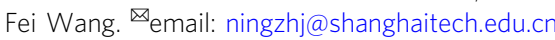


alide perovskites are rising as star materials for nextgeneration solar cells. However, the state of the art lead perovskite still needs to be upgraded since: firstly, the heavy metal character of lead may cause an environmental concern $^{1,2}$; secondly, according to Shockley-Queisser limitation, the highest efficiency of lead perovskite is $<31 \%$ due to its large bandgap $(1.55 \mathrm{eV})^{3}$. The development of alternative perovskite materials is therefore highly desirable. Tin perovskite is regarded as an ideal candidate due to its narrow bandgap and environmental benign character ${ }^{4}$. Up to now, tin perovskite solar cell (PSC) demonstrates the highest efficiency among all kinds of lead-free perovskites ${ }^{5}$.

Despite the rapid development of tin PSCs, the efficiency is much lower than lead perovskite nowadays. One critical factor is the extremely low open-circuit voltage. High defect density in the film is one important factor contributing to the low voltage ${ }^{6}$. Some methods are implemented to reduce defect density of tin perovskite, e.g., addition of reductive additives ${ }^{7-9}$, and perovskite dimensionality manipulation ${ }^{10-12}$, which increased the voltage of the device from $0.3 \mathrm{~V}$ to $0.6 \mathrm{~V}$ (ref. ${ }^{13}$ ). However, compared to its bandgap $(1.35 \mathrm{eV})$, the voltage loss of tin PSCs is still over $0.7 \mathrm{~V}$, much larger than that of lead perovskite. It is highly desirable to clarify the mechanism for the low voltage of tin PSCs and find solutions.

Another possible factor for the low voltage of tin PSCs could be the deep Lowest Unoccupied Molecular Orbital (LUMO) energy level of electron transporting layers (ETLs), e.g., $[6,6]$-phenyl- $\mathrm{C}_{61}$-butyric acid methyl ester $(\mathrm{PCBM})^{14,15}$ and buckminsterfullerene $\left(\mathrm{C}_{60}\right)^{16-18}$, which limited the maximum attainable photovoltage $\mathrm{e}^{19}$.

Here, we introduce indene- $\mathrm{C}_{60}$ bisadduct (ICBA) as ETL for tin PSCs to replace the generally used PCBM. ICBA with shallower energy level brings a larger maximum attainable voltage and it suppresses iodide remote doping that reduces interface carriers recombination. As a result, the use of ICBA as ETL improves the voltage of the device to $0.94 \mathrm{~V}$, which is significantly higher than that of the device using PCBM as ETL $(0.6 \mathrm{~V})$. The device based on ICBA demonstrates an unprecedentedly high efficiency of $12.4 \%$, which is almost $30 \%$ higher than the highest efficiency reported up to now ${ }^{20-22}$. Moreover, the device shows excellent shelf stability by maintaining $90 \%$ of the initial efficiency for over 3800 hours. This work clarifies the mechanism for the low voltage of tin PSCs, and presents a strategy to increase the voltage and efficiency of tin PSCs.

\section{Results}

Perovskite film structure. Perovskite films are prepared using a typical anti-solvent method via a one-step process (details can be found in Methods section). We tune the concentration of PEA $\left(\mathrm{PEA}=\mathrm{C}_{6} \mathrm{H}_{5} \mathrm{CH}_{2} \mathrm{CH}_{2} \mathrm{NH}_{3}{ }^{+}\right)$in precursors to get the $\mathrm{PEA}_{x}$ $\mathrm{FA}_{1-x} \mathrm{SnI}_{3}\left(\mathrm{FA}=\mathrm{HC}\left(\mathrm{NH}_{2}\right)_{2}{ }^{+}\right)$perovskites (abbreviated as PEA $x$, $x=0$ and 15). Films are prepared on poly(3, 4-ethylenedioxythiophene):poly(styrene sulfonate) (PEDOT) ${ }^{18}$ substrates to control the nucleation and orientation of perovskite. $\mathrm{NH}_{4} \mathrm{SCN}$ (SCN) is used as additive to modify perovskite film growth.

We characterized the crystal structure by X-ray diffraction (XRD). The diffraction peaks of the perovskite film with $\mathrm{NH}_{4} \mathrm{SCN}$ (PEA15-SCN) show a higher intensity (Fig. 1a) and a smaller fullwidth half-maximum (FWHM) value (Fig. 1b), indicating a larger domain size for PEA15-SCN film.

We then studied the microstructure of perovskite film by grazing-incidence wide-angle X-ray scattering (GIWAXS). The strong diffraction spot in 90 degree shows that PEA15-SCN film is perpendicularly grown on the substrate along [100] direction $^{23,24}$ (Supplementary Figs. 2-4). Both films with and without SCN grown on PEDOT show similar diffraction spots from single layer and double layer structures at both small and large grazing-incidence angles (Supplementary Fig. 3). This is completely different from the image of hierarchy structure grown on $\mathrm{NiO}_{\mathrm{X}}$ substrate ${ }^{12}$. We conclude that low dimensional structures homogeneously distribute in the film, which can be ascribed to the higher binding energy of PEA and FA molecules on PEDOT substrate, as calculated by density functional theory (DFT) simulation (Supplementary Fig. 6, Supplementary Table 1).

We performed spectroscopy characterization to study the structure of the films further. The absorption edge of the film based on PEA15-SCN $(905 \mathrm{~nm})$ is quite close to that of threedimensional (3D) structure PEA0 (917 nm; Supplementary Fig. 7a), implying the presence of 3D-like structures in the film. In combination with GIWAXS measurement, it can be speculated that part of PEA molecules are consumed for constructing twodimensional (2D) and 2D-like structures, which are mixed with 3D structures in the film (Supplementary Fig. 8). The absorption spectrum of PEA15-SCN film demonstrates a lower Urbach energy of $\sim 65 \mathrm{meV}$ (Fig. 1c), which indicates the reduced defect concentration with the addition of $\mathrm{NH}_{4} \mathrm{SCN}$ (refs. ${ }^{25,26}$ ). Scanning electron microscope (SEM) images of PEA15 and PEA15SCN films are similar and both of them show smooth morphology (Fig. 1d).

Device structure design. We measured the energy levels of the perovskite films for device structure design. Based on ultraviolet photoelectron spectroscopy (UPS), the Fermi level and valence band minimum for PEA15-SCN perovskite film (Fig. 2a) are calculated to be $-4.52 \mathrm{eV}$ and $-5.08 \mathrm{eV}$, respectively. Combining the optical bandgaps of $1.39 \mathrm{eV}$ for PEA15-SCN determined by Tauc plots (Supplementary Fig. 7b), the conduction band maximum of perovskite film is calculated to be $-3.69 \mathrm{eV}$ for PEA15SCN (Fig. 2b). Considering the shallow conduction band position of tin perovskite, the typically used ETL, such as PCBM shows a large energy level offset due to its much deeper band position. Since the maximum attainable photovoltage is determined by the quasi-Fermi level splitting, $V_{\mathrm{OC}}=\frac{1}{q}\left(E_{\mathrm{Fn}}-E_{\mathrm{Fp}}\right)$, the use of shallower energy level ETL could increase the $V_{\text {OC }}$ of the device ${ }^{27}$. Therefore, we used ICBA as ETL, since it has a LUMO level of $-3.74 \mathrm{eV}$ shallower than the commonly used PCBM of $-3.91 \mathrm{eV}$ (ref. ${ }^{28}$ ).

Device performance. We fabricated the device based on PEA15SCN films above using ICBA and PCBM as ETL, respectively. The device based on ICBA achieved a much-enhanced voltage up to $0.94 \mathrm{~V}$ (Fig. 3a). The efficiency certified in an independent lab is up to $12.4 \%\left(V_{\mathrm{OC}}=0.94 \mathrm{~V}, J_{\mathrm{SC}}=17.4 \mathrm{~mA} \mathrm{~cm}^{-2}, \mathrm{FF}=75 \%\right.$; Table 1, Supplementary Fig. 9). This is $\sim 30 \%$ higher than the best value reported up to now ${ }^{20-22}$. In contrast, the device based on PCBM shows much worse efficiency of $7.7 \%$ (Fig. 3b, Supplementary Fig. 10), and the voltage is only $0.60 \mathrm{~V}$. The device based on ICBA shows a low hysteresis as the current density-voltage $(J-V)$ curves under reverse and forward scan overlapped well (Fig. 3b). The integrated photocurrent density $\left(17.3 \mathrm{~mA} \mathrm{~cm}^{-2}\right)$ obtaining from the external quantum efficiency (EQE) spectra of PEA15-SCN device (Fig. 3c) agrees closely with current density $\left(J_{\mathrm{SC}}\right)$ in the $J-V$ curves. Moreover, the device performance shows good reproducibility (Fig. 3d, Supplementary Fig. 11).

We then fabricated devices based on PEA15 and ICBA for comparison, which shows $V_{\mathrm{OC}}$ of $0.78 \mathrm{~V}$, much smaller than that the film with SCN (Table 1). Despite the current density of the film is comparable to that based on PEA15-SCN, the overall efficiency of the device is only $10.1 \%$. 

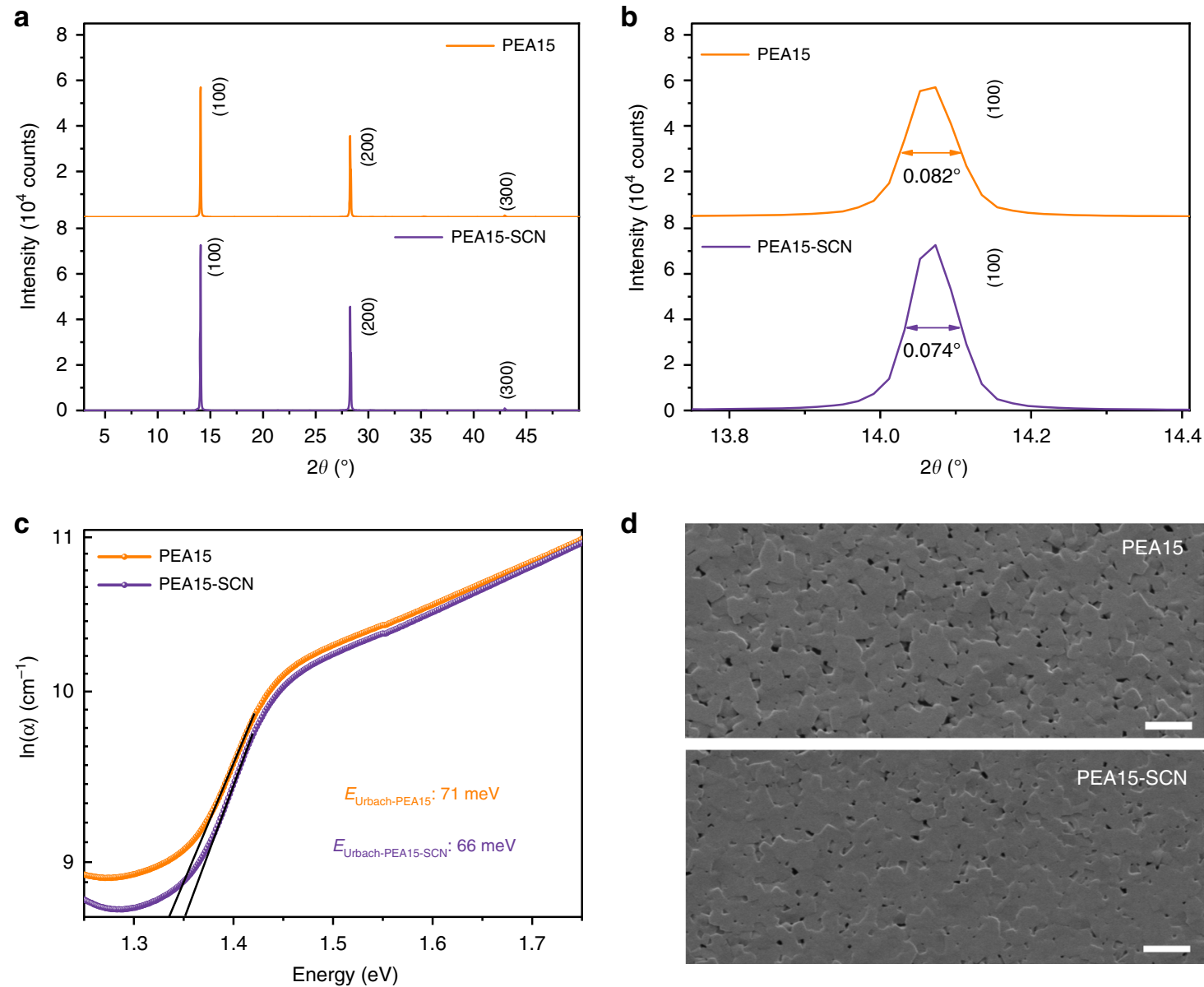

d
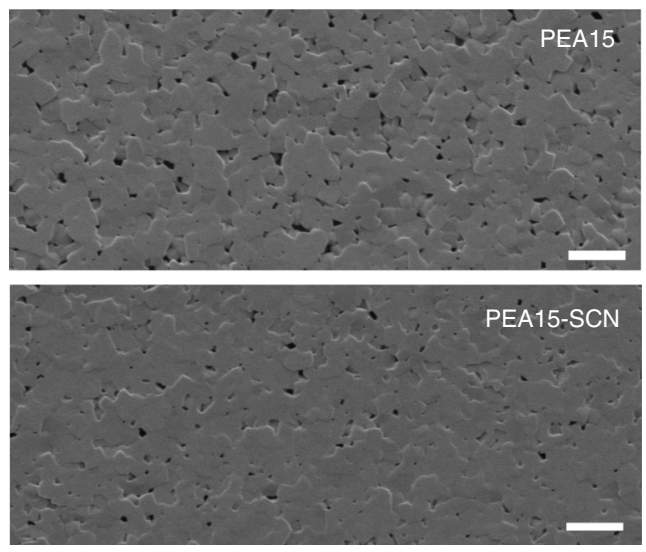

Fig. 1 Perovskite film characterization. a XRD spectra of perovskite films and $\mathbf{b}$ the corresponding FWHM values of (100) peak. c The Urbach energy of perovskite films. d SEM images of perovskite films. The scale bar is $1 \mu \mathrm{m}$.

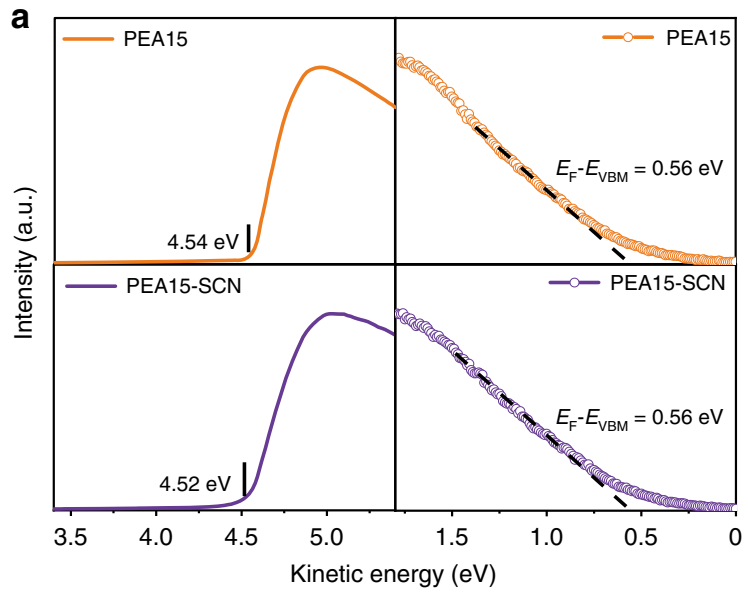

b

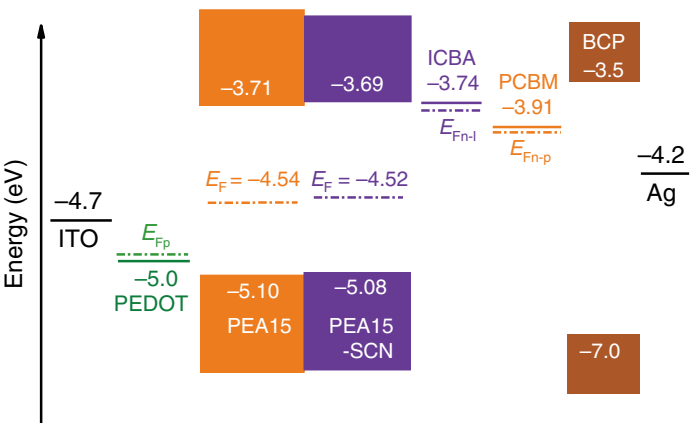

Fig. 2 Band structure of tin PSCs. a UPS spectra of secondary electron cutoff and valence band of perovskite films. b Schematic illustration of energy levels. Dashed lines represent the quasi-Fermi level of ICBA $\left(E_{\mathrm{Fn}-1}\right), \operatorname{PCBM}\left(E_{\mathrm{Fn}-\mathrm{P}}\right)$, and $\operatorname{PEDOT}\left(E_{\mathrm{Fp}}\right)$.

The PEA15-SCN device shows good shelf stability: the encapsulated device maintained $90 \%$ of the initial performance for as long as 3800 hours (Fig. 3e). Steady-state power conversion efficiency (PCE) measurement was carried out to evaluate the operation stability of the device. The device showed stable efficiency at continuous operation at maximum power point for $150 \mathrm{~s}$ (Fig. 3f). However, under longer time illumination, the efficiency decay slows, and the device loses $50 \%$ of its initial performance after continuous operation for over $300 \mathrm{~min}$ under amplitude modulation (AM) 1.5 illumination (Supplementary Fig. 13). The efficiency decay might be ascribed to the ion migration or accumulation of carriers at the interface.

Device characterization. To clarify the mechanism for the muchenhanced voltage and performance of the device based on ICBA, we studied the interface recombination between perovskite and ETL. Firstly, scanning Kelvin probe microscopy (SKPM) was 
a

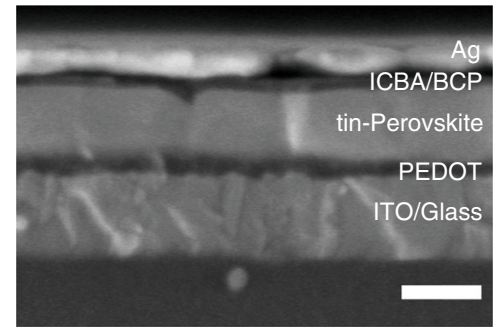

d

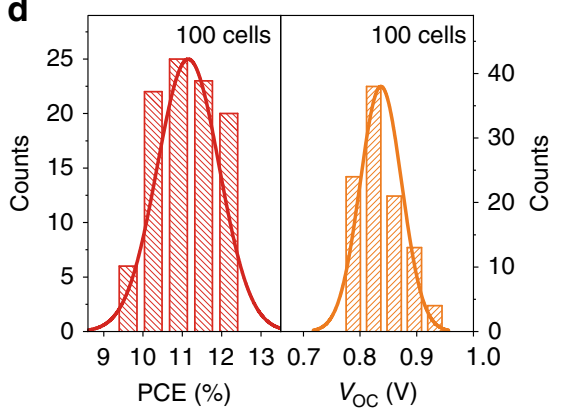

b

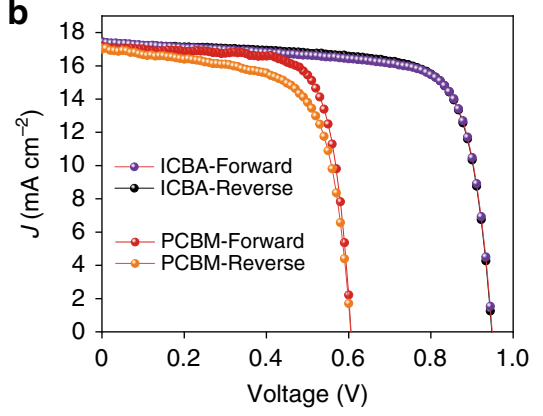

e

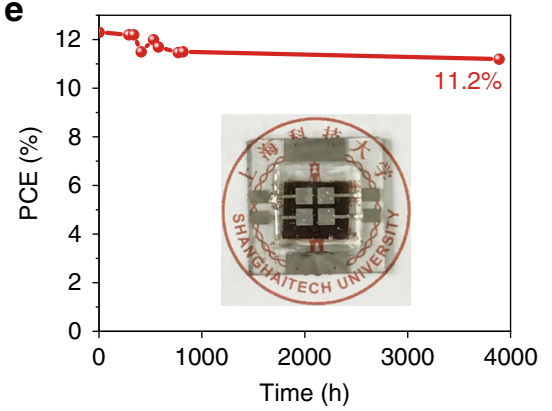

c

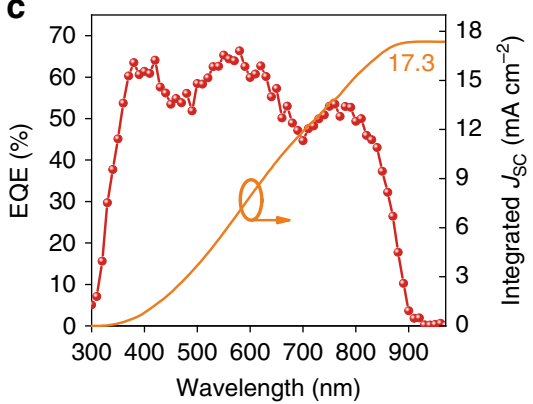

f

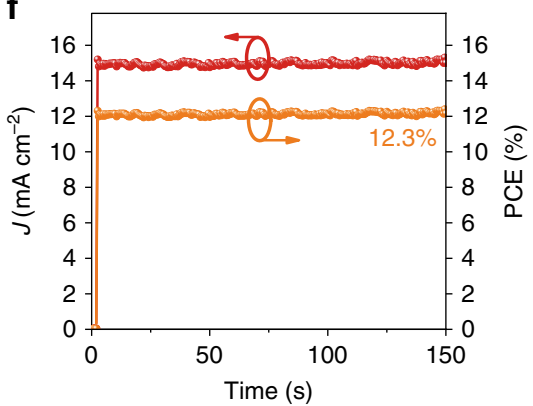

Fig. 3 Photovoltaic performances of tin PSCs. a Cross-section SEM image of PEA15-SCN device. The scale bar is $200 \mathrm{~nm}$. $\mathbf{b} \mathrm{J}-\mathrm{V}$ curves of the certified PEA15-SCN device with ICBA and champion device of PEA15-SCN film with PCBM. c EQE curve and integrated $J_{S C}$ of the certified PEA15-SCN device. d Histograms for PCE and $V_{O C}$ of PEA15-SCN device. e The stability of encapsulated PEA15-SCN device stored in $\mathrm{N}_{2}$ atmosphere. $\mathbf{f}$ Stabilized power output for the PEA15-SCN device (at $0.81 \mathrm{~V}$ ) under simulated AM $1.5 \mathrm{G}$ solar illumination at $100 \mathrm{~mW} \mathrm{~cm}-2$.

\begin{tabular}{|c|c|c|c|c|}
\hline Device & $\begin{array}{l}\text { PCE } \\
(\%)\end{array}$ & $\begin{array}{l}V_{\text {oc }} \\
\text { (V) }\end{array}$ & $\begin{array}{l}J_{S C} \\
\left(m A c^{-2}\right)\end{array}$ & $\begin{array}{l}\mathbf{F F} \\
(\%)\end{array}$ \\
\hline PEDOT /PEA15/ICBA & 10.1 & 0.78 & 17.8 & 72 \\
\hline $\begin{array}{l}\text { PEDOT/PEA15-SCN/ } \\
\text { PCBM }\end{array}$ & 7.7 & 0.60 & 17.1 & 74 \\
\hline $\begin{array}{l}\text { PEDOT/PEA15-SCN/ } \\
\text { ICBA }\end{array}$ & 12.4 & 0.94 & 17.4 & 75 \\
\hline
\end{tabular}

employed to analyze interfacial energy level alignment of perovskite/ETL interface (Fig. 4a, Supplementary Figs. 14 and 15$)^{29}$. The surface potential of ICBA is $20 \mathrm{mV}$ higher than perovskite, indicating that the Fermi level of ICBA is $20 \mathrm{meV}$ higher than that of perovskite. Similarly, we deduced that the Fermi level of PCBM is $\sim 100 \mathrm{meV}$ higher than perovskite (Fig. 4a). Hence, it can be calculated that the Fermi level of PCBM is $80 \mathrm{meV}$ higher than that of ICBA. Considering that the LUMO level of PCBM is deeper than ICBA (Fig. 4b), the higher Fermi level of PCBM indicates that more states are occupied by electrons, i.e., it has higher electron density ${ }^{27}$. This can be attributed to remote doping from iodide, which can act as donor to inject electrons into ETL $\left(\right.$ ref. $\left.{ }^{30}\right)$. In contrast, the shallow LUMO level of ICBA prohibits electron injection from iodide, giving rise to less electron density. The existence of iodide in ETL can be ascribed to ion migration in perovskite film ${ }^{30}$.

The high electron density of PCBM could aggravate interface carrier recombination with $\mathrm{p}$-type tin perovskite film $\mathrm{m}^{31}$. We hence performed time-resolved photoluminescence (TRPL) decay measurement to test the interface carriers recombination. The TRPL of different perovskite films shows a bi-exponential decay (Fig. 4c), including a fast component $\left(\tau_{1}\right)$, and a slow component $\left(\tau_{2}\right)^{32}$ (Table 2). The fast component can be ascribed to the rapid carriers recombination across the interfaces of perovskite and transporting laye ${ }^{33}$. The perovskite film contacting PCBM shows a smaller $\tau_{1}$, indicating faster carriers recombination at the interface. We used electroluminescence (EL) measurement under bias to estimate the interface recombination further ${ }^{34,35}$. As shown in Fig. 4d, PEA15-SCN device with ICBA shows an obvious EL peak, while extremely weak EL peak is observed for device with PCBM. The much higher EL intensity shows that the interface recombination is significantly suppressed, agreeing well with TRPL characterization.

We then used a model to quantitatively calculate the voltage loss due to interface carrier recombination. The $V_{\mathrm{OC}}$ loss $\left(\Delta V_{\mathrm{OC}}^{\text {nrad }}\right)$ of non-radiative decay at perovskite/ETL interface can be estimated by external radiative efficiency (ERE) from EL measurement based on the following equation ${ }^{36}$ :

$$
\Delta V_{\mathrm{OC}}^{\mathrm{nrad}}=\frac{-k_{\mathrm{B}} T}{q} \ln \mathrm{ERE}
$$

Where ERE is the electroluminescent EQE of the device. Based on the ERE of PEA15 and PEA15-SCN (Supplementary Fig. 16), the $\Delta V_{\mathrm{OC}}^{\mathrm{nrad}}$ are determined to be $417 \mathrm{mV}$ and $226 \mathrm{mV}$ for the device based on PCBM and ICBA, respectively. Hence, we conclude that interface recombination is another important factor that responsible for the low voltage of the device based on PCBM.

To investigate the function of the addition of $\mathrm{NH}_{4} \mathrm{SCN}$ in the film to device performances, we performed transient photovoltage (TPV) and transient photocurrent (TPC) measurements to test the density of defect states in the bandgap directly ${ }^{37}$ (Fig. 4e). The film with $\mathrm{NH}_{4} \mathrm{SCN}$ shows less density of defect states inside the bandgap, especially in the region close to the conduction band. We tested $V_{O C}$ versus illumination intensity curves (Fig. $4 \mathrm{f}$ ) and found that slope for the device based on PEA15-SCN is smaller than that based on PEA15, indicating the decrease of recombination of the film. Furthermore, the low Urbach energy of PEA15SCN film in absorption spectra indicates the decrease of defect density as well. The reduced defect density of the film can be 

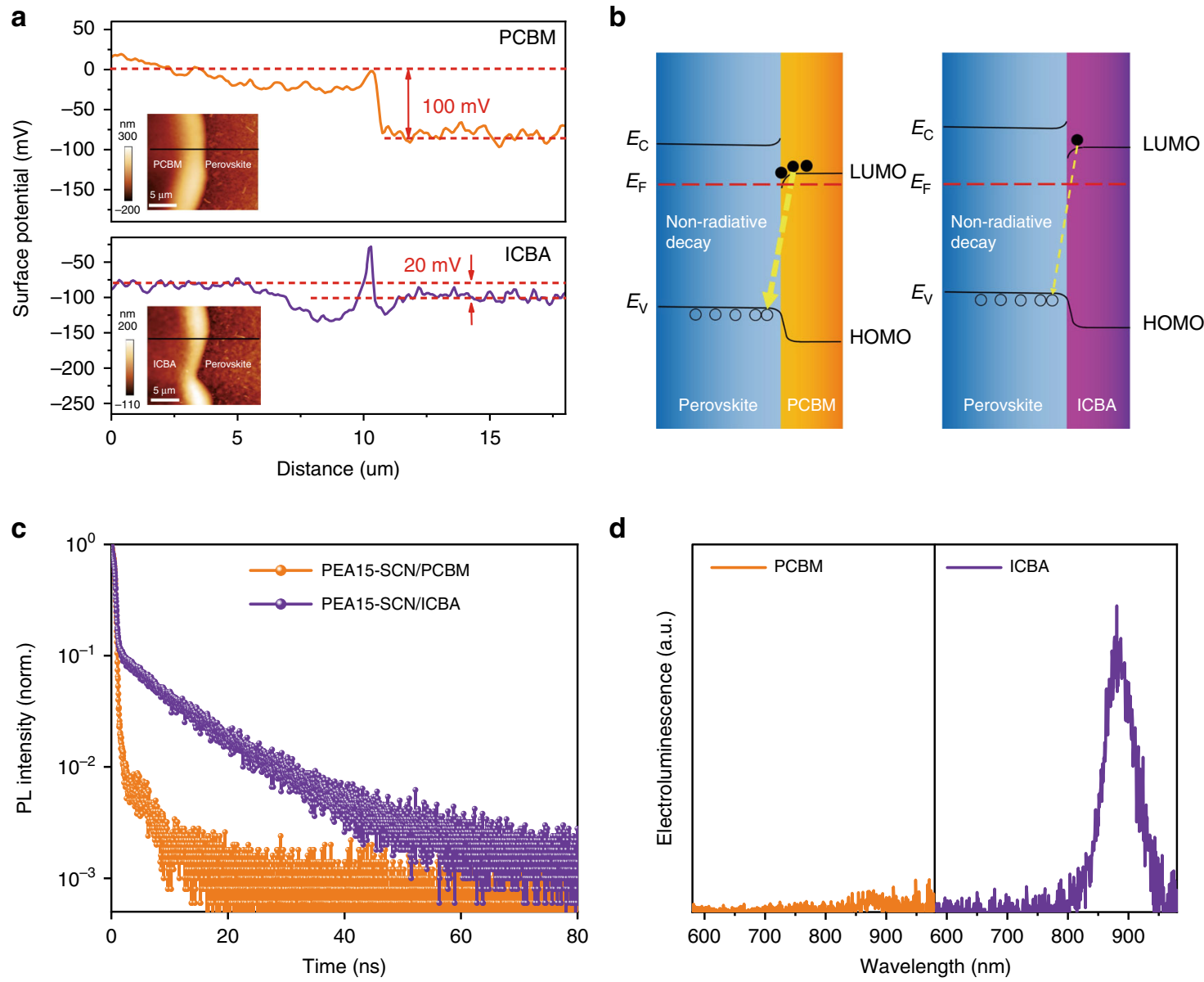

d
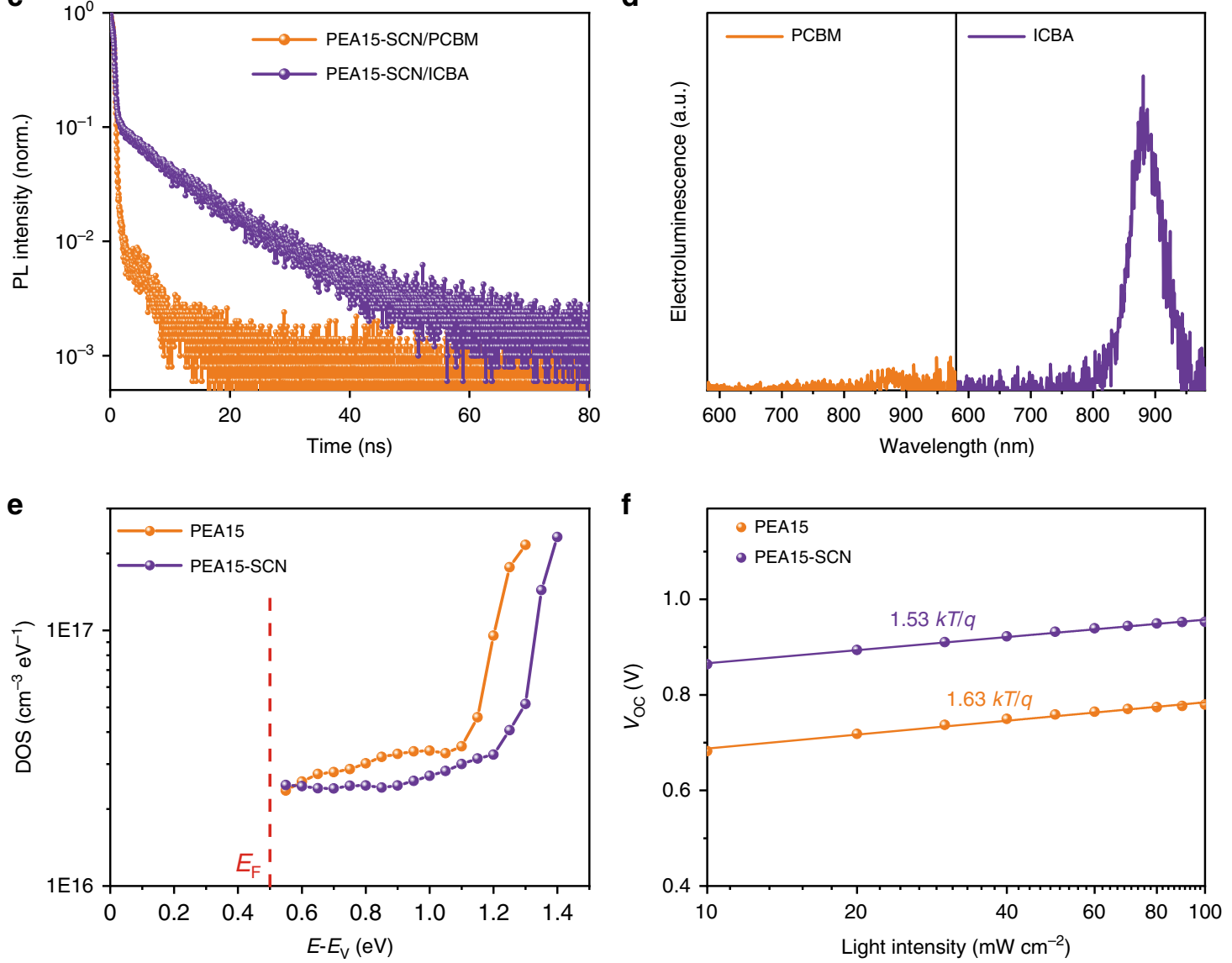

Fig. 4 Recombination and defect density characterization. a Surface potential distribution of PEA15-SCN/PCBM and PEA15-SCN/ICBA from SKPM measurement. The insert images are AFM topography images for the corresponding samples. $\mathbf{b}$ The schematic diagrams of interface recombination for the two samples. c Time-resolved photoluminescence kinetics at $840 \mathrm{~nm}$ for the ITO/PEDOT/perovskite/ETL films after encapsulation. $\mathbf{d}$ Electroluminescence spectra of perovskite films under bias voltage of $2 \mathrm{~V}$. e The density of states in the bandgap calculated from TPV and TPC. $\mathbf{f} V_{O C}$ versus illumination intensity for the devices.

ascribed to the increase of domain size that reduced the grain boundary of the film, as discussed above. The reduced density of defects of SCN-treated film brings increased voltage and efficiency of the device.

\section{Discussion}

In this work, we develop a device structure of tin PSCs using ICBA as ETL. The higher LUMO energy level of ICBA improves the maximum attainable voltage of the device and reduces the remote doping caused interface recombination. Furthermore, the use of $\mathrm{NH}_{4} \mathrm{SCN}$ as additive and PEDOT as hole transporting layer for perovskite growth reduces the defect density of the film. As a result, we are able to realize an unprecedentedly high voltage of $0.94 \mathrm{~V}$ for tin PSCs. Finally, we achieve a certified PCE up to $12.4 \%$, which is almost $30 \%$ higher than the highest efficiency reported. With the open-circuit voltage approaching its theoretic limitation, this work indicates a huge potential for efficiency improvement of tin PSCs. 
Table 2 Decay time of PEA15-SCN film with ICBA and PCBM.

\begin{tabular}{llc} 
& $\boldsymbol{\tau}_{\mathbf{1}}$ (ns) & $\boldsymbol{\tau}_{\mathbf{2}}$ (ns) \\
\hline ICBA & 1.1 & 12.1 \\
PCBM & 0.25 & 2.2 \\
\hline
\end{tabular}

\section{Methods}

Device fabrication. $\mathrm{SnI}_{2}$, formamidinium iodide (FAI), phenethylammonium iodide (PEAI), and $\mathrm{NiO}_{\mathrm{X}}$ were prepared according to literatures ${ }^{12,13}$. N,N-dimethylformamide (DMF), dimethyl sulfoxide (DMSO), chlorobenzene, isopropyl alcohol, $\mathrm{SnF}_{2}$, and $\mathrm{NH}_{4} \mathrm{SCN}$ were purchased from Sigma-Aldrich. Cleaned indium tin oxide (ITO) glass was treated in an ultraviolet ozone (UVO) machine for $20 \mathrm{~min}$ before fabrication. The PEDOT (Heraeus-Clevios P VP AI 4083) solution was spin coated onto ITO substrate at $6000 \mathrm{rpm}$ for $60 \mathrm{~s}$ and then annealed at $140{ }^{\circ} \mathrm{C}$ for $20 \mathrm{~min}$. A $0.8 \mathrm{M}$ perovskite precursor (PEAI:FAI:SnI $2: \mathrm{SnF}_{2}=0.15: 0.85: 1: 0.075$ ) and with or without $\mathrm{NH}_{4} \mathrm{SCN}(5 \mathrm{~mol} \%)$ were added in mixed solvent (DMF:DMSO = $4: 1 \mathrm{~V} / \mathrm{V}$ ) and stirred at $70^{\circ} \mathrm{C}$ for $1 \mathrm{~h}$. The precursor was spin coated at $1000 \mathrm{rpm}$ and $5000 \mathrm{rpm}$ for $10 \mathrm{~s}$ and $30 \mathrm{~s}$, respectively. And $600 \mu \mathrm{L}$ toluene (Sinopharm Chemical Reagent Co. Ltd., redistilled) was dropped during the second process at eighth second. The substrate was then annealed at $70^{\circ} \mathrm{C}$ for $10 \mathrm{~min}$. The films of PEA15 and PEA0 were made by the same procedure. A total of $18 \mathrm{mg} \mathrm{mL}^{-1}$ ICBA (1Material) or PCBM (nano-C) in chlorobenzene was spin coated at $1000 \mathrm{rpm}$ for $30 \mathrm{~s}$, and then annealed at $70^{\circ} \mathrm{C}$ for $10 \mathrm{~min}$. Saturated bathocuproine in isopropyl alcohol was spin coated at $6000 \mathrm{rpm}$ for $30 \mathrm{~s}$, followed by annealing at $70^{\circ} \mathrm{C}$ for $10 \mathrm{~min}$. All precursors were filtered with $0.22 \mu \mathrm{m}$ polytetrafluoroethylene (PTFE) filters before spin coated. Finally, $100 \mathrm{~nm}$ Ag layer was deposited under vacuum of $<10^{-7}$ torr using an Angstrom Engineering deposition system.

Film characterizations. XRD was recorded on a Bruker D8 Advance using $\mathrm{Cu} \mathrm{Ka}$ source $(\lambda=1.54 \AA)$. PL spectra were carried out by the exciting wavelength at $480 \mathrm{~nm}$ of spectrofluorometer (Fluorolog; HORIBA FL-3) with a standard $450 \mathrm{~W}$ Xenon CW lamp. TRPL spectroscopy at $840 \mathrm{~nm}$ was measured using Fluorolog HORIBA FL-3 with a pulsed source at $504 \mathrm{~nm}$ DeltaDiode DD-510L. The UV-vis spectra of the perovskite films deposited on PEDOT were recorded by UV-vis spectrophotometer (Agilent cary5000). SEM images were recorded on JSM-7800. UPS was performed for films on PEDOT using a Thermo Fisher ESCALAB 250XI, and the samples were transferred from glovebox to vacuum chamber with a portable transfer capsule. Curve fitting was performed using the Thermo Avantage software. The curves were corrected based on the C1s peak at $284.8 \mathrm{eV}$. GIWAXS studies were performed at the BL16B1 beamline of Shanghai Synchrotron Radiation Facility (SSRF), Shanghai, China, using beam energy of $10 \mathrm{keV}(\lambda=1.24 \AA)$ and a Mar 225 detector. The grazing-incidence angles for all films were $0.2^{\circ}$ and $1.0^{\circ}$, respectively. GIXGUI Matlab toolbox was utilized for necessary corrections of GIWAXS raw patterns and collecting the azimuth angle ${ }^{38}$.

Device characterization. $J-V$ curves were measured using a Keithley 2400 source unit under simulated AM $1.5 \mathrm{G}$ solar illumination at $100 \mathrm{~mW} \mathrm{~cm}^{-2}$ (1 sun). The light intensity was calibrated by means of a KG-5 Si diode with a solar simulator (Enli Tech, Taiwan). The devices are measured in reverse scan $(1.0-0 \mathrm{~V}$, step $0.01 \mathrm{~V})$ and forward scan $(0-1.0 \mathrm{~V}$, step $0.01 \mathrm{~V})$ with a delay time of $30 \mathrm{~ms}$. The $J-V$ curves for all devices were measured by masking the active area using a metal mask with an area of $0.04 \mathrm{~cm}^{2}$. The steady-state PCE was performed at $0.81 \mathrm{~V}$. Devices were stored and tested in the same nitrogen-filled glovebox (Vigor).

The EQE spectra were measured by a commercial system (Solar cell scan 100 Beijing Zolix Instruments Co., Ltd). The cells were subjected to monochromatic illumination (150 W Xe lamp passing through a monochromator and appropriate filters). The light intensity was calibrated by a standard photodetector (QE-B3/ S1337-1010BQ, Zolix). The light beam was chopped at $180 \mathrm{~Hz}$, and the response of the cell was acquired by a Stanford Research SR830 lock-in amplifier.

TPV and TPC measurements were carried out using a setup comprising a $532 \mathrm{~nm}$ wavelength laser to provide steady-state bias light, a $640 \mathrm{~nm}$ wavelength laser, and an oscilloscope. A power-adjustable $532 \mathrm{~nm}$ wavelength laser was used to get a steady $V_{\mathrm{OC}}$ of the device. A $640 \mathrm{~nm}$ diode laser was used to modulate the $V_{\mathrm{OC}}$ on top of a constant light bias. The pulse duration was set to $1 \mu$ s and the repetition rate to $50 \mathrm{~Hz}$ by the function generator of the oscillator. The digital oscilloscope recorded the data induced by the light perturbation, using $1 \mathrm{M} \Omega$ input impedance for the TPV measurement and $50 \Omega$ impedance for TPC measurement. We determined the charge generated $(\Delta Q)$ in the devices by integrating the TPC curve by the $640 \mathrm{~nm}$ laser pulse without $532 \mathrm{~nm}$ light bias present. The calculated $C=$ $\Delta Q / \Delta V_{\mathrm{OC}}$ result is the capacitance. The total charge carrier was obtained by integrating the $C$ versus $V_{\mathrm{OC}}$. The carrier concentration $(n)$ for each open-circuit voltage was calculated by dividing each charge carriers with the device volume. The density of states (DOS) in the mid-gap can be obtained by differentiating the carrier density with respect to the $V_{\text {OC }}$ following a previously reported procedure ${ }^{37}$.

The current density-luminance-radiance $(J-V-R)$ characteristics were measured by a Keithley 2400 source meter, and a fiber integrating sphere (FOIS-1) couple with a QE Pro 650 spectrometer (Ocean Optics). The devices were tested on top of the integrating sphere, and only forward light emission could be collected All device test processes were carried out in the $\mathrm{N}_{2}$-filled glovebox.

First-principles calculations. First-principles calculations were performed within the framework of DFT using plane-wave pseudopotential methods, as implemented in the Vienna Ab-initio Simulation Package ${ }^{39}$. The generalized gradient approximation formulated by Perdew, Burke, and Ernzerhof was used as the exchange-correlation functional. The electron-core interactions were described by the projector augmented-wave ${ }^{40}$ method for the pseudo potentials. The cutoff energy for the plane-wave basis set used to expand the Kohn-Sham orbitals was $400 \mathrm{eV}$. The Gamma-centered $k$-point mesh with a grid spacing of $2 \pi \times 0.03 \AA^{-1}$ was used for electronic Brillouin zone integration. For adsorption energy calculation, the vacuum thickness was set to be $20 \AA$. The equilibrium structural parameters (including both lattice parameters and internal coordinates) of each involved bulk material were obtained via total energy minimization by using the conjugate gradient algorithm, with the force convergence threshold of $0.01 \mathrm{eV} \AA^{-1}$. for slab structures, only the internal coordinates are relaxed. The optB86b-vdW (ref. ${ }^{41}$ ) functional is adopted throughout the whole calculation.

SKPM measurement. The amplitude-modulation SKPM was operated combined with a Cypher S atomic force microscopy (AFM; Asylum Research, Oxford Instruments) and a HF2LI Lock-in amplifier (Zurich Instruments) in $\mathrm{N}_{2}$-filled glovebox. The resonance frequency $\omega_{0}$ and spring constant of AFM conducting tips are $\sim 127 \mathrm{kHz}$ and $5.0 \mathrm{Nm}^{-1}$, respectively.

Tin PSC certification at SIMIT (Shanghai, China). The device certification tests were performed at an independent lab (SIMIT, Chinese Academy of Sciences). And SIMIT is accredited by China National Accreditation Service for Conformity Assessment (CNAS) to ISO/IEC 17025 and by the International Laboratory Accreditation Cooperation (ILAC) Mutual Recognition Arrangement.

A silicon reference solar cell (PVM1211, NREL_ISO tracking\#:1974) was used to set the irradiance at $100 \mathrm{~mW} \mathrm{~cm}^{-2}$ at standard testing conditions in accordance with IEC 60904-3 ed.2 AM 1.5 G. J-V characteristics of tin PSCs were measured under simulated sunlight by steady-state class AAA solar simulator according to IEC 60904-9 ed.2. The spectral mismatch was calculated and mismatch correction was performed according to IEC 60904-7 ed.3. The $J-V$ curves were measured in forward and reverse scans with a scanning speed of $90 \mathrm{mV} \mathrm{s}^{-1}$.

Encapsulation method. Devices were encapsulated by quartz glass and UV-glue for $J-V$, EQE, TRPL, TPV, and TPC measurements. Films were double sealed by plastic bags before XRD, SEM, PL, UV-vis, and GIWAXS measurements.

Reporting summary. Further information on experimental design is available in the Nature Research Reporting Summary linked to this paper.

\section{Data availability}

The data that support the findings of this study are available from the corresponding author upon reasonable request.

Received: 16 June 2019; Accepted: 12 February 2020;

Published online: 06 March 2020

\section{References}

1. Abate, A. J. J. Perovskite solar cells go lead free. Joule 1, 659-664 (2017).

2. Yu, D. et al. Stability improvement under high efficiency-next stage development of perovskite solar cells. Sci. China Chem. 62, 684-707 (2019).

3. Correa-Baena, J. P. et al. Promises and challenges of perovskite solar cells. Science 358, 739-744 (2017).

4. Shi, Z. et al. Lead-free organic-inorganic hybrid perovskites for photovoltaic applications: recent advances and perspectives. Adv. Mater. 29, 1605005 (2017).

5. Diau, E. W.-G., Jokar, E. \& Rameez, M. Strategies to improve performance and stability for tin-based perovskite solar cells. ACS Energy Lett. 4, 1930-1937 (2019)

6. Ke, W. \& Kanatzidis, M. G. Prospects for low-toxicity lead-free perovskite solar cells. Nat. Commun. 10, 965 (2019).

7. Marshall, K. P., Walker, M., Walton, R. I. \& Hatton, R. A. Enhanced stability and efficiency in hole-transport-layer-free CsSnI3 perovskite photovoltaics. Nat. Energy 1, 16178 (2016)

8. Lee, S. J. et al. Fabrication of efficient formamidinium tin iodide perovskite solar cells through SnF2-pyrazine complex. J. Am. Chem. Soc. 138, 3974-3977 (2016).

9. Tai, Q. et al. Antioxidant grain passivation for air-stable tin-based perovskite solar cells. Angew. Chem. Int. Ed. 58, 806-810 (2019). 
10. Qiu, J., Xia, Y., Chen, Y. \& Huang, W. Management of crystallization kinetics for efficient and stable low-dimensional Ruddlesden-Popper (LDRP) lead-free perovskite solar cells. Adv. Sci. 6, 1800793 (2018).

11. Shao, S. et al. Enhancing the crystallinity and perfecting the orientation of formamidinium tin iodide for highly efficient Sn-based perovskite solar cells. Nano Energy 60, 810-816 (2019).

12. Wang, F. et al. 2D-quasi-2D-3D hierarchy structure for tin perovskite solar cells with enhanced efficiency and stability. Joule 2, 2732-2743 (2018).

13. Liao, Y. et al. Highly oriented low-dimensional tin halide perovskites with enhanced stability and photovoltaic performance. J. Am. Chem. Soc. 139, 6693-6699 (2017).

14. Liu, X., Wang, Y. B., Xie, F. X., Yang, X. D. \& Han, L. Y. Improving the performance of inverted formamidinium tin iodide perovskite solar cells by reducing the energy-level mismatch. ACS Energy Lett. 3, 1116-1121 (2018).

15. Kayesh, M. E. et al. Coadditive engineering with 5 -ammonium valeric acid iodide for efficient and stable Sn perovskite solar cells. ACS Energy Lett. 4, 278-284 (2018).

16. Liao, W. et al. Lead-free inverted planar formamidinium tin triiodide perovskite solar cells achieving power conversion efficiencies up to $6.22 \%$. Adv. Mater. 28, 9333-9340 (2016).

17. Zhao, Z. et al. Mixed-organic-cation tin iodide for lead-free perovskite solar cells with an efficiency of 8.12\%. Adv. Sci. 4, 1700204 (2017).

18. Shao, S. et al. Highly reproducible sn-based hybrid perovskite solar cells with 9\% efficiency. Adv. Energy Mater. 8, 1702019 (2018).

19. Nishikubo, R., Ishida, N., Katsuki, Y., Wakamiya, A. \& Saeki, A. Minute-scale degradation and shift of valence-band maxima of $(\mathrm{CH} 3 \mathrm{NH} 3) \mathrm{SnI} 3$ and $\mathrm{HC}$ (NH2)2SnI3 perovskites upon air exposure. J. Phys. Chem. C 121, 19650-19656 (2017).

20. Jokar, E., Chien, C. H., Tsai, C. M., Fathi, A. \& Diau, E. W. G. Robust tinbased perovskite solar cells with hybrid organic cations to attain efficiency approaching 10\%. Adv. Mater. 31, 1804835 (2019).

21. Ran, C. et al. Conjugated organic cations enable efficient self-healing FASnI3 solar cells. Joule 3, 3072-3087 (2019).

22. $\mathrm{Wu}$, T. et al. Efficient and stable tin-based perovskite solar cells by introducing $\pi$-conjugated Lewis base. Sci. China Chem. 63, 107-115 (2019).

23. Tsai, H. et al. High-efficiency two-dimensional Ruddlesden-Popper perovskite solar cells. Nature 536, 312 (2016)

24. Byun, J. et al. Efficient visible quasi-2D perovskite light-emitting diodes. $A d v$. Mater. 28, 7515-7520 (2016).

25. Urbach, F. J. P. R. The long-wavelength edge of photographic sensitivity and of the electronic absorption of solids. Phys. Rev. 92, 1324 (1953).

26. De Wolf, S. et al. Organometallic halide perovskites: sharp optical absorption edge and its relation to photovoltaic performance. J. Phys. Chem. Lett. 5, 1035-1039 (2014).

27. Lin, Y. et al. Matching charge extraction contact for wide-bandgap perovskite solar cells. Adv. Mater. 29, 1700607 (2017).

28. He, Y. J., Chen, H. Y., Hou, J. H. \& Li, Y. F. Indene-C60 bisadduct: a new acceptor for high-performance polymer solar cells. J. Am. Chem. Soc. 132, 1377-1382 (2010).

29. Zhang, M. et al. Reconfiguration of interfacial energy band structure for highperformance inverted structure perovskite solar cells. Nat. Commun. 10, 4593 (2019).

30. Zhao, T., Chueh, C.-C., Chen, Q., Rajagopal, A. \& Jen, A. K. Y. Defect passivation of organic-inorganic hybrid perovskites by diammonium iodide toward highperformance photovoltaic devices. ACS Energy Lett. 1, 757-763 (2016).

31. Wolff, C. M. et al. Reduced interface-mediated recombination for high opencircuit voltages in CH3NH3PbI3 solar cells. Adv. Mater. 29, 1700159 (2017).

32. Shi, D. et al. Solar cells. Low trap-state density and long carrier diffusion in organolead trihalide perovskite single crystals. Science 347, 519-522 (2015).

33. Stranks, S. D. et al. Electron-hole diffusion lengths exceeding 1 micrometer in an organometal trihalide perovskite absorber. Science 342, 341-344 (2013).

34. Jiang, Q. et al. Surface passivation of perovskite film for efficient solar cells Nat. Photon. 13, 460-466 (2019).

35. Luo, D. et al. Enhanced photovoltage for inverted planar heterojunction perovskite solar cells. Science 360, 1442-1446 (2018).

36. Krückemeier, L., Rau, U., Stolterfoht, M. \& Kirchartz, T. How to report record open-circuit voltages in lead-halide perovskite solar cells. Adv. Energy Mater. 10, 1902573 (2019).

37. Ip, A. H. et al. Hybrid passivated colloidal quantum dot solids. Nat. Nanotechnol. 7, 577-582 (2012).
38. Jiang, Z. GIXSGUI: a MATLAB toolbox for grazing-incidence X-ray scattering data visualization and reduction, and indexing of buried three-dimensional periodic nanostructured films. J. Appl. Crystallogr. 48, 917-926 (2015).

39. Kresse, G. \& FurthmüllerJ. J. Pr. B. Efficient iterative schemes for ab initio total-energy calculations using a plane-wave basis set. Phys. Rev. B 54, 11169 (1996).

40. Blochl, P. E. Projector augmented-wave method. Phys. Rev. B 50, 17953-17979 (1994).

41. Klimeš, J., Bowler, D. R. \& Michaelides, A. Van der Waals density functionals applied to solids. Phys. Rev. B 83, 195131 (2011).

\section{Acknowledgements}

The authors gratefully acknowledge financial support from the National Key Research and Development Program of China (under grant nos. 2016YFA0204000 and 2016YFA0200700), ShanghaiTech start-up funding, 1000 young talent program, National Natural Science Foundation of China (61935016, U1632118, 21571129, 21625304, and 21875280), Shanghai key research program (16JC1402100), and Centre for Highresolution Electron Microscopy (CћEM), SPST, ShanghaiTech University under contract no. EM02161943. The authors appreciate the high-performance computing (HPC) Platform of ShanghaiTech University. The authors appreciate the BL16B1 beamline of Shanghai Synchrotron Radiation Facility (SSRF), Shanghai, China. The authors appreciate Dr. Na Yu, Rong Gao, and the Instrument Analysis Center of ShanghaiTech University.

\section{Author contributions}

X.J. and F.W. contributed equally to this work. X.J., F.W., and Z.N. designed and directed the study. X.J. and F.W. contributed to all the experimental work. Q.W. carried out and interpreted the DFT studies. H.L. performed the PL, TRPL, and XRD measurements. Y.S helped to carry out GIWAXS, and EL measurements and data analysis. W.Z. supervised the EQE and TPV measurements. P.C. supervised the UPS measurement. C.W., Q.C., and L.C. conducted the SKPM measurement and data analysis. Z.N. supervised the whole project. X.J., F.W., and Z.N write the manuscript. All authors discussed the results and commented on the final manuscript.

\section{Competing interests}

The authors declare no competing interests.

\section{Additional information}

Supplementary information is available for this paper at https://doi.org/10.1038/s41467020-15078-2.

Correspondence and requests for materials should be addressed to Z.N

Peer review information Nature Communications thanks Ashraful Islam and the other, anonymous, reviewer(s) for their contribution to the peer review of this work.

Reprints and permission information is available at http://www.nature.com/reprints

Publisher's note Springer Nature remains neutral with regard to jurisdictional claims in published maps and institutional affiliations.

Open Access This article is licensed under a Creative Commons Attribution 4.0 International License, which permits use, sharing, adaptation, distribution and reproduction in any medium or format, as long as you give appropriate credit to the original author(s) and the source, provide a link to the Creative Commons license, and indicate if changes were made. The images or other third party material in this article are included in the article's Creative Commons license, unless indicated otherwise in a credit line to the material. If material is not included in the article's Creative Commons license and your intended use is not permitted by statutory regulation or exceeds the permitted use, you will need to obtain permission directly from the copyright holder. To view a copy of this license, visit http://creativecommons.org/ licenses/by/4.0/.

(C) The Author(s) 2020 